\title{
Laboratorio de comunicación estratégica para el empleo. Análisis de 16 años de experiencias desde el Servicio de Empleo de la Universidad de Alicante
}

\author{
Rosa María TORRES VALDÉS \\ Universidad de Alicante \\ rosa.torres@ua.es
}

Recibido: $11 / 10 / 2012$

Aceptado: 23/01/2013

\begin{abstract}
Resumen
Este trabajo abarca el periodo 1994-2010 y presenta el análisis de un compendio de investigaciones que contemplaban interrogantes acerca de la comunicación universitaria en materia de empleo de sus egresados. Se inicia con estudios exploratorios del estado de la cuestión tanto en el ámbito teórico como empresarial, y de información universitaria en los mass media, todo ello a fin de obtener información útil que permita diseñar estrategias, experimentarlas, evaluar resultados y obrar en consecuencia. Eran y siguen siendo tantos los actores sociales implicados o públicos, las situaciones y coyunturas y las variables de influencia que la proyección en el tiempo para investigar y actuar era una necesidad imperiosa, para que las propuestas de protocolos de comunicación pudieran evaluarse y adaptarse a los constantes cambios del escenario socioeconómico. Refleja el esfuerzo por considerar la variable comunicación como elemento estratégico del Gabinete de Iniciativas Para el Empleo (GIPE), pudiendo ser este un caso de estudio.
\end{abstract}

Palabras clave: comunicación estratégica, relaciones públicas, empleo, desarrollo socioeconómico.

\section{Strategic Communication Laboratory for Employment. Analysis of 16 years of Experiences from the Employment Service of the University of Alicante}

\begin{abstract}
This work covers the period 1994-2010, and shows an analysis of communication research on university employment. It starts with exploratory studies about the state of the art both in theory and in business and media, with the purpose of obtaining useful information in order to designing communication strategies, implement them, evaluate impact and act accordingly with the results. Situation, circumstances, stake holders involved and variable of influence were (and remain) so, a research action approach that was a need. This article reflects the effort to consider the communication as a strategic element for the carrier office of the university (GIPE) which could be considered a case of study.

Keywords: strategic communication, public relations, employment, socioeconomic development.

\section{Referencia normalizada}

TORRES VALDÉS, Rosa María (2013): “Laboratorio de comunicación estratégica para el empleo. Análisis de 16 años de experiencias desde el Servicio de Empleo de la Universidad de Alicante". Estudios sobre el mensaje periodístico. Vol. 19. Núm. especial abril, págs.: 1049-1056. Madrid, Servicio de Publicaciones de la Universidad Complutense.
\end{abstract}

Sumario: 1. Introducción. Fuentes y estado de la cuestión. 2. Metodología. 3. Desarrollo. 4. Resultados. 5. Conclusiones. 6. Referencias bibliográficas. 7. Referencias documentales.

\section{Introducción. Fuentes y estado de la cuestión}

Este análisis de las experiencias recogidas durante 16 años en el Servicio de Empleo de la Universidad de Alicante que vamos a presentar es un tema de importancia socioeconómica, máxime en el contexto dual que vivimos actualmente. Por una parte, 
estamos inmersos en una crisis económica y de confianza que afecta negativamente al empleo y a la creación de empresas; por otra, el Espacio Europeo de Educación Superior y la estrategia Universidad 2015 reconoce el «papel relevante que ha de jugar la Universidad en Europa en los próximos años, tanto como agente clave en la consolidación de una economía sostenible y responsable basada en el conocimiento, como institución de educación superior fundamental para mejorar la formación de la sociedad y facilitar su adaptación a los rápidos cambios existentes en los mercados de trabajo» (Estrategia Universidad 2015, 2010: web).

Ante la responsabilidad de crear un Servicio Universitario de Empleo (SUE), se impone la necesidad de reflexionar en torno posibles variables de influencia en los procesos de mejora del futuro empleo de los universitarios. En el marco de esta reflexión, era necesario considerar la comunicación como vector clave en la estrategia de reducción de las disonancias $u d$ supra mencionadas, y de aproximación realista entre los subsistemas Empresa y Universidad. Y todo ello desde la más profunda humildad investigadora, sin pretender agotar las posibilidades de análisis que cabría realizar sobre numerosos aspectos estructurales y coyunturales de la economía, en nuestro caso, alicantina.

Introducidos ya en este campo, la realidad comenzó a mostrarnos que dilucidar la cuestión comunicativa en la estrategia para el empleo universitario iba a ser un viaje largo, de exploración, para descubrir, describir y ensayar, orientándonos al futuro sin abandonar la mirada retrospectiva, a fin de comparar, corregir y continuar según las lecciones aprendidas. Todo aconsejaba abordar el trabajo emprendiendo investigaciones paralelas, multidisciplinares, utilizando diferentes técnicas convergentes, conformando lo que denominamos «Investigación Puzle». De sus resultados pretendíamos extraer conclusiones de soporte a la gestión y toma de decisiones (muchas de ellas vinculadas a la estrategia relacional y comunicativa) en el recién creado servicio universitario de empleo que en la Universidad de Alicante se denominó GIPE: Gabinete de Iniciativas Para el Empleo.

Asumida la proyección longitudinal y transversal de la investigación, desde el inicio y en todo momento consideramos imprescindible servirnos de las valiosas aportaciones de investigadores del área socioeconómica, de psicología social y comunicación, además de seguir la información institucional tanto de la Comisión Europea como de las políticas de Gobierno nacional y regional. Incluimos también la información procedente de los mass media relacionada con la Universidad y el Empleo (fundamentalmente noticias y ofertas de empleo). De este modo alcanzábamos dos de los objetivos propuestos: por una parte, inferir los primeros razonamientos, generadores de una serie de interrogantes fundamentales para definir con claridad el problema de investigación, los objetivos y plantear las posibles hipótesis de partida y el diseño metodológico. Por otra, nos permitía observar la evolución y los cambios sociotécnicos globales que se han ido produciendo y la evolución del servicio universitario de empleo con relación a dichos cambios (1994-2010).

Así pues, las fuentes de referencia a lo largo de este período han sido contribuciones de trabajos previos procedentes de diversos ámbitos. En el ámbito de la Comunicación y las Relaciones Públicas, se tomó como referencia la teoría de los «sistemas interpenetrantes» (Grunig y Hunt, 2000: 114) desarrollada por Preston y Post. Estos 
autores conciben «las organizaciones y sistemas en su entorno como sistema interpenetrantes que ni controlan completamente al entorno social ni son controlados por él» (Grunig y Hunt, 2000: 114), es decir, su conexión con el concepto de «vínculos» (Esman, 1972, en Grunig y Hunt, 2000: 230-232) relacionando «vínculos» y «consecuencias». La revisión realizada por Grunig de teorías y modelos, haciendo hincapié en la Teoría situacional para identificar públicos, sirvió para dibujar el mapa de públicos del GIPE. Por otra parte, se tuvo en cuenta el modelo de los sujetos (Noguero, 1990:113), cuyo interés radica en el análisis conceptual que realiza el autor con relación a los sujetos de las relaciones públicas, clasificándolos en promotores, ejecutores y receptores, estos últimos tipificados como públicos (internos y externos).

Hasta hace relativamente poco, en el ámbito de educación superior -empleo y desarrollo socioeconómico- las fuentes de información sobre las relaciones entre educación superior y mundo laboral eran más bien escasas, teniendo que recurrir a estadísticas oficiales, con criterios distintos según el organismo y zona geográfica, no pudiendo explotar el potencial real de dichas estadísticas. Sin embargo, en 2003, el Consejo de Coordinación Universitaria del Ministerio de Educación organizó el seminario «Métodos de análisis de la inserción laboral de los universitarios», en la Universidad de León. Cabe destacar en este ámbito el análisis de Andrés Pedreño Muñoz «Universidad: utopías y realidades, 1994-1997», y los resultados del documento de trabajo del Instituto Interuniversitario de Economía Internacional (IEI) de la Universidad de Alicante sobre «Formación, atracción y generación de talentos y capital humano». La relación del empleo con el desarrollo local parecía una cuestión lógica y nos interesó desde el primer momento. En este caso recurrimos a los trabajos sobre desarro1lo, redes e innovación de Antonio Vázquez Barquero (recopilación de 1983-1999) y a las aportaciones de Ismael Blanco y Ricard Gomá sobre las ideas de gobierno de proximidad y redes participativas para innovación y desarrollo.

Entre las actuales contribuciones del ámbito de la comunicación que han sido consultadas (recordemos que es un trabajo realizado durante 16 años), destacamos las Actas del I Congreso Internacional Latina de Comunicación Social (2009), fundamentalmente las que se refieren a perfiles profesionales, prácticas y Espacio Europeo de Educación superior, como es el caso de «Prácticas en empresas para los estudiantes de Periodismo dentro del EEES, nuevos retos para los COIES de las Universidades y su adaptación a Bolonia» (Bellido y Sánchez, 2009: web); «El entrenamiento en técnicas creativas en el Espacio Europeo de Educación Superior» (Jiménez Martín y Moncholi, 2009: web); «UEMCOM, prácticas académicas dentro del EEES» (Lemrini, Moreno-Baquerizo y Rodríguez García, 2009: web) y, finalmente, «Modelando futuros profesionales de la comunicación: la incorporación de la educación en valores a los currícula universitarios» (Piñeiro, 2009: web).

Actas del I Congreso de la Asociación Española de Investigación de la comunicación (Santiago de Compostela, enero de 2008). Es justo reconocer que todos los contenidos han sido enormemente útiles y satisfactorios por dos razones fundamentales: primero porque la lectura de todas las contribuciones evidenció que lo que al inicio del servicio universitario de empleo -en nuestro caso- partía de un enfoque intuitivo sobre la necesidad de investigar la comunicación, no era desacertado; segundo, por- 
que muestra la pertinencia de la investigación en comunicación dentro del escenario de desarrollo socioeconómico vinculado de la relación universidad-empresa, que es en definitiva el objeto de este trabajo. En este sentido, cabe destacar de la «Sesión especial. Investigación de la comunicación y docencia en las facultades de comunicación» (Reguero Jiménez, 2008: web) las aportaciones de García Galindo (Universidad de Málaga), con su propuesta de establecer «nuevos ejes de estudio en función de los cambios sociales, tecnológicos y empresariales» y la de Petxo Idoyaga (Universidad del País Vasco), con su referencia a las «Prácticas de producción creativas para facilitar la integración de los alumnos a un entorno laboral cambiante y polivalente...». Por su parte Jacques Guyot (Universidad París VIII) alude a «oportunidades profesionales». La experiencia de «Unimonitor» aportada por Bárbara Mazza (Universidad Sapienza de Roma) es, sin duda, una de las líneas con las que más converge nuestra investigación, dado que se trata del observatorio de la formación y el trabajo, aunque en su caso solo sea dentro del ámbito de la comunicación. La mención de una red estatal que dialoga con instituciones y empresas tiene una gran trascendencia en el tema que abordamos. Dado que el GIPE se ocuparía de la inserción laboral en todas titulaciones prestando especial atención a la dificultad de inserción laboral de jóvenes científicos, otra fuente relevante la formaron las declaraciones de los Congresos de Comunicación Social de la Ciencia, ${ }^{1}$ desde la declaración de Granada en 1999: «La información científica es una fecundísima semilla para el desarrollo social, económico y político de los pueblos. La complicidad entre los científicos y el resto de los ciudadanos es una excepcional celebración de la democracia»; hasta la declaración de Pamplona en marzo de 2010: «Los tiempos de crisis siempre han sido el momento de analizar cómo avanzar hacia el futuro, con la creación de nuevos modelos de desarrollo. Desde el mundo de la comunicación de la ciencia ofrecemos nuestro compromiso en este reto: una nueva cultura en la que todos somos necesarios».

\section{Metodología}

A través de este estudio pretendemos explorar y describir las posibles disonancias del binomio universidad-empresa, contrastando la percepción que cada uno de los actores tiene del otro. La voluntad de todo ello es diseñar protocolos de comunicación estratégica para la intervención en procesos de inserción laboral de los distintos perfiles de titulados de la Universidad de Alicante, sin perder de vista ni la estructura ni la coyuntura de la economía alicantina.

A continuación se describe un problema ${ }^{2}$ de relación universidad-empresa, y de gestión de la comunicación para la promoción del empleo y autoempleo universitario.

1 Para el trabajo que nos ocupa, estas declaraciones, ayer y hoy, encajan en la definición del problema descrito de la relación universidad-empresa, y nuevamente nos encontramos el papel de la información y la comunicación como clave en procesos de desarrollo sostenible y democrático.

2 En nuestro caso, el problema del empleo se planteó en 1994, sin embargo destacamos la actualidad del tema y la importancia que cobra indagar sobre el papel de comunicación estratégica en el ámbito del empleo dentro del marco de la actual crisis económica y de confianza. 
El Servicio Universitario de Empleo tiene la función poner en contacto a empresarios, responsables políticos, académicos, alumnos y titulados. Es decir, relaciona a todos los ciudadanos, lo que otorga un rol de intermediación y dinamización que exige capacidad de comunicación estratégica.

Para alcanzar los objetivos señalados suele optarse por el método de la investigación exploratoria y descriptiva, además de prescriptiva. ${ }^{3}$ Por una parte se recurre a la producción intelectual existente en los ámbitos estudiados y se describen las realidades observadas en relación con el objeto de estudio; por otra, se recurre a la investigación acción o Action Research (AR), y se desarrolla un análisis cualitativo ex post facto y cuantitativo.

\section{Desarrollo}

Dado que el trabajo abarca el período 1994-2010, y a fin de no interrumpir el correlato, la información para este apartado recoge por fases el compendio de investigaciones y protocolos de comunicación elaborados a partir de los resultados de las mismas. Dichas fases son: Fase I. Septiembre 1994-Diciembre 1995 (Fase inicial de trabajo con la apertura del servicio); Fase II A (1996-1999); Fase II B (1997-2002); Fase III (2003-2010); Metodología y Resultados. Por tanto, se exponen en anexos en formato de tablas.

\section{Resultados}

En este trabajo se presentan dos tipos de resultados: los correspondientes al compendio de investigaciones propiamente dichas por un lado y, por otro, los protocolos de comunicación surgidos de los hallazgos y conclusiones de investigación. Todo ello se encuentra recogido en tablas en los siguientes anexos:

- ANEXO 1: Tabla «Análisis a efectos de retroalimentación del proceso comunicativo relacionado con el empleo universitario I». A continuación se incluye «Protocolos de comunicación estratégica y relaciones públicas diseñados en relación con resultados de Fase 1 de investigación». Le siguen «Tabla de Fase IIA, IIB y III», tituladas «Análisis a efectos de retroalimentación del proceso comunicativo relacionado con el empleo universitario».

- ANEXO 2: Gráficas de resultados de investigación «Fase 1 (1994-1994) sobre la visión de las empresas sobre la universidad y los universitarios» y «Opinión de los ciudadanos en relación con la universidad». Después está la gráfica de «Diferenciales de percepción de las empresas hacia la universidad como formador para el empleo», producto de estudio de dos fases, 1997-1998 y 1999-2000.

- ANEXO 3: Protocolos más importantes de los elaborados a partir de los procesos de investigación acción.

- Protocolo 2: Comunicación interna y externa basada en el modelo de los sujetos.

- Protocolo 3: Comunicación y relaciones públicas con agencias de desarrollo local.

- ANEXO 4: Cuadro de herramientas de comunicación estratégica para servicios de empleo universitarios y similares.

3 Término relacionado con la investigación acción. 


\section{Conclusiones}

En relación con los objetivos de investigación planteados, se exponen las siguientes conclusiones.

Los trabajos de la primera fase nos permitieron confirmar que la identificación de las disonancias del binomio universidad-empresa era contrastable con la percepción por parte de la empresa de la universidad como entidad formadora más en la teoría que en la práctica. Se atribuye porque la universidad, en el diseño curricular de sus titulaciones, no contempla como objetivo prioritario las necesidades de la empresa.

Como consecuencia de este diagnóstico se diseñaron protocolos de comunicación estratégica y se experimentó con su puesta en práctica, pudiendo constatar que contribuyeron a una intervención más eficaz en los procesos de inserción laboral de los distintos perfiles de titulados de la Universidad de Alicante. Así, se dio a conocer el valor de los mismos y el papel de promotor de desarrollo que podía jugar la universidad en la estructura y coyuntura de la economía alicantina.

Además de las reuniones de expertos, el estudio sobre factores de excelencia empresarial y potencialidades de empleabilidad en el subsistema de empresas demandantes de prácticas aportaron información objetiva y rigurosa sobre las condiciones de empleo de los egresados de la Universidad de Alicante en el área de influencia de la misma.

Se pudo tipificar la información aparecida en los mass media en relación con la universidad, así como el tipo de oferta de empleo que podía entenderse como cualificada, lo que permitió confirmar que el modelo de comunicación utilizado por la universidad era de tipo informativo y técnico. De este modo, a menos que se tuviera formación en el tema, la comprensión era difícil, reforzando la percepción de distancia con la empresa y la sociedad. Así, pudo tipificarse la oferta de empleo y el perfil demandado, salvo para casos de I+D, en los que no se definía claramente. En todos los casos, la experiencia era un mérito que rara vez podía alcanzar un recién titulado.

La investigación acción permitió la observación por ciclos de avance progresivo y la consecución de objetivos. Tales logros pudieron medirse a través de criterios cuantificables e interpretables, como:

- Número de peticiones de información tanto desde la universidad como desde fuera por parte de instituciones, otras universidades, empresas...

- Grado de satisfacción de los usuarios del servicio, tanto alumnos como titulados y empresas.

- Número de invitaciones a participar en actos de otras entidades.

- Respuesta mayoritaria del conjunto de servicios de empleo universitario de las universidades españolas a la llamada a la reunión de expertos.

- Número de patrocinios y de acciones de mecenazgo conseguidas.

- Número de personas en prácticas y contratadas, y evolución de la demanda.

- Número de proyectos europeos y cuantía económica a partir de las relaciones establecidas.

- Número de noticias en los medios de comunicación con informaciones orientadas a los objetivos de puesta en valor de la universidad y los universitarios. 
El conjunto de protocolos permitió alcanzar el objetivo de elaborar una sencilla herramienta de consulta (Anexo 5) para la gestión de comunicación estratégica con el público de servicios de empleo universitarios y similares. Se presenta en forma de un cuadro que recoge las estrategias, tácticas, técnicas e instrumentos que siempre pueden utilizarse de forma práctica y objetiva.

La estrategia metodológica basada en la combinación de técnicas cuantitativas y cualitativas, así como las fuentes secundarias fiables consultadas, nos ha permitido fotografiar el fenómeno informativo comunicativo en el ámbito del empleo universitario, y desarrollar el análisis de 16 años de trabajo continuado, con la debida compatibilización multi e interdisciplinar presente en el problema de investigación.

En el momento actual, cabe repensar la comunicación desde la universidad para generar confianza en el perfil de profesionales que forma, y proyectarse como institución catalizadora junto con otras instituciones de desarrollo socioeconómico. El hecho de probar los protocolos expuestos en este trabajo a fin de valorar si se producen resultados similares puede ser una línea de investigación que se abre a partir de esta humilde contribución a mostrar la pertinencia de la comunicación en procesos de empleo y autoempleo universitario.

\section{Referencias bibliográficas}

BLANCO, Ismael y GOMÁ, Ricard (coords., 2002): Gobiernos Locales y Redes Participativas. Barcelona, Ariel Social.

GRUNIG, James y HUNT, Todd (2000): Dirección de Relaciones Públicas Barcelona, Gestión 2000.

NOGUERO, Antonio (1995): Función social de las Relaciones. Colección Comunicación y Relaciones Públicas. Barcelona, PPU.

PEDREÑO MUÑOZ, Andrés (1998): Universidad: utopias y realidades. Universidad de Alicante, 1994-1997. Madrid, Civitas.

TORRES VALDÉS, Rosa María; UNDIEDT LECUONA, Carlos; y PARDO CASADO, Mario (2009): Formación, atracción y generación de talentos y capital humano. Documento de trabajo del Instituto Interuniversitario de Economía Internacional (IEI). DT 2009-06. Alicante, Servicio de Publicaciones de la Universidad de Alicante.

VÁZQUEZ BARQUERO, Antonio (1999): Desarrollo, redes e innovación. Lecciones sobre desarrollo endógeno. Madrid, Pirámide.

VIDAL GARCÍA, Javier (2003): Métodos y análisis de la inserción laboral de los universitarios. Consejo de coordinación universitaria. Ministerio de Educación, Cultura y Deporte. León, Secretariado de Publicaciones de la Universidad de León.

\section{Referencias documentales}

ACTAS DEL CONGRESO ASOCIACIÓN ESPAÑOLA DE INVESTIGACIÓN DE LA COMUNICACIÓN (AE-IC): http://www.ae-ic.org/santiago2008/contents/esp /comunicaciones_detf6fe.html?id_seccio $=3 \&$ id_apartat $=9$ 
ACTAS DEL CONGRESO COMUNICACIÓN SOCIAL DE LA CIENCIA: http://www.parqueciencias.com/parqueciencias/quienes_somos/declaracion-granada.html www.muncyt.es/stfls/MUNCYT/Publicaciones/prensacongreso.pdf

ACTAS DEL CONGRESO SOCIEDAD LATINA DE COMUNICACIÓN SOCIAL: http://www.revistalatinacs.org/09/Sociedad/00_indexBBB.html

ASOCIACIÓN DE AGENTES DE DESARROLLO LOCAL: http://www.aprodelmadrid.org

ASOCIACIÓN ESPAÑOLA DE INVESTIGADORES EN COMUNICACIÓN: http://www.ae-ic.org

COMISIÓN EUROPEA: http://ec.europa.eu/

FUNDACIÓN CONOCIMIENTO Y DESARROLLO: http://www.fundacioncyd.org /wps/portal/

INSTITUTO INTERUNIVERSITARIO DE ECONOMÍA INTERNACIONAL (IEI): http://www.iei.ua.es

INTERNATIONAL PUBLIC RELATIONS ASSOCIATION: http://www.ipra.org/

MINISTERIO DE CIENCIA E INNOVACIÓN: http://www.micinn.es/

MINISTERIO DE EDUCACIÓN: http://www.educacion.es

MINISTERIO DE TRABAJO: http://www.mtin.es/

\section{Rosa María TORRES VALDÉS}

Universidad de Alicante

Departamento de Comunicación y Psicología Social

Profesora Asociada (Comunicación Audiovisual y Publicidad)

rosa.torres@ua.es 